\title{
Engineering gold nanoantennae to enhance the emission of quantum emitters
}

\author{
Mario Agio, ${ }^{a}$ Giorgio Mori, ${ }^{a}$ Franziska Kaminski, ${ }^{a}$ Lavinia Rogobete, ${ }^{a}$ Sergei Kühn, ${ }^{a}$ \\ Victor Callegari, ${ }^{b}$ Philipp M. Nellen, ${ }^{b}$ Franck Robin, ${ }^{c}$ Yasin Ekinci, ${ }^{d}$ \\ Urs Sennhauser, ${ }^{b}$ Heinz Jäckel, ${ }^{c}$ Harun H. Solak, ${ }^{d}$ and Vahid Sandoghdar ${ }^{a}$ \\ ${ }^{a}$ Laboratory of Physical Chemistry, ETH Zurich, 8093 Zurich, Switzerland; \\ ${ }^{b}$ Electronics/Metrology/Reliability Laboratory, EMPA, \\ Swiss Federal Laboratories for Materials Testing and Research, 8600 Dübendorf, Switzerland; \\ ${ }^{c}$ Communication Photonics Group, Electronics Laboratory (IfE), ETH Zurich, \\ 8092 Zurich, Switzerland; \\ ${ }^{d}$ Laboratory for Micro and Nanotechnology, Paul Scherrer Institute, \\ 5232 Villigen PSI, Switzerland
}

\begin{abstract}
We study the fluorescence enhancement of a single emitter coupled to two spherical gold nanoparticles and discuss the differences with respect to coupling to a single one. We also show that by changing the aspect ratio of the nanoparticles we can easily tune the plasmon-mediated enhancement from the infrared to the visible range. We present the fabrication of our nanoantennae by two alternative methods, namely X-ray interference lithography followed by focused ion beam milling and electron beam lithography. The manufactured structures are characterized individually by confocal microscopy.
\end{abstract}

Keywords: Fluorescence enhancement, metal nanoparticle, optical nanoantenna, localized surface plasmon resonance, spectroscopy

\section{INTRODUCTION}

Noble metal nanoparticles $(\mathrm{NPs})^{1,2}$ are finding several interesting applications in nanoscience. For example, they can be employed to enhance fluorescence, ${ }^{3-7}$ to increase nonlinear processes like two-photon absorption ${ }^{8}$ or frequency mixing, ${ }^{9}$ they can be used to generate sub-diffraction limited laser spots, ${ }^{10}$ as sensors,${ }^{11}$ and for surfaceenhanced Raman scattering. ${ }^{12,13}$ Metal NPs exhibit a strong electromagnetic response at optical wavelengths thanks to the existence of localized surface plasmon resonances, ${ }^{1,2}$ hence the term optical nanoantenna. ${ }^{14}$ Such name fits particularly well to the problem of fluorescence enhancement where, in analogy to a conventional antenna, the NP favors absorption and radiation of electromagnetic energy by a load, in this situation represented by a single quantum emitter.

While some research groups have studied fluorescence enhancement by detecting an ensemble average of several NPs and emitters, ${ }^{15,16}$ we have chosen to investigate a simpler system composed by a single molecule coupled to a single gold NP to allow a more quantitative comparison with the electromagnetic theory of fluorescence enhancement. ${ }^{5}$ We have experimentally confirmed that the fluorescence signal is directly proportional to the local electric field intensity and to the quantum efficiency that the emitter exhibits when coupled to the NP. While the near field increases when the emitter approaches the gold NP, the quantum efficiency decreases up to a point that the emitter is quenched. ${ }^{5}$ The decrease in the quantum efficiency is due to the energy dissipated

Further author information: (Send correspondence to M.A.)

M.A.: E-mail: mario.agio@phys.chem.ethz.ch, Telephone: +41 (0)44 6323322.

F.K.: Present address: Niels Bohr Institute, 2100 Copenhagen, Denmark.

S.K.: Present address: School of Engineering, University of California at Santa Cruz, 95064 Santa Cruz, CA, U.S.A..

P.M.N: Present address: RUAG Aerospace, 8304 Wallisellen, Switzerland.

Y.E.: Present address: Laboratory of Metal Physics and Technology, ETH Zurich, 8093 Zurich, Switzerland.

Optomechatronic Micro/Nano Devices and Components III, edited by Lixin Dong, Yoshitada Katagiri,

Eiji Higurashi, Hiroshi Toshiyoshi, Yves-Alain Peter, Proceedings of SPIE Vol. 6717, 67170R, (2007)

0277-786X/07/\$18 - doi: $10.1117 / 12.754367$

Proc. of SPIE Vol. 6717 67170R-1 
by the metal. ${ }^{17}$ Because of these competing phenomena, achieving very large fluorescence enhancements is not possible with single spherical gold NPs. To circumvent this issue we have numerically studied and designed non-spherical NPs that exhibit a very high quantum efficiency even when the emitter is in close proximity of the nanoantenna. ${ }^{18}$ We want to apply these ideas to realize an experiment where the fluorescence enhancement is much stronger than what was found in ensemble measurements and for single NPs. For this reason, we will try to adapt our ideal designs to situations accessible to experiments with single molecules.

The paper is organized as follows. In Sec. 2 we discuss the problem of fluorescence enhancement in general. We then focus on a single emitter coupled to two NPs, first spheres and second spheroids. We conclude the section by designing gold nanoantennae made of two spheroids resonant at wavelengths around 600-650 nm. In Sec. 3 we discuss in some detail the fabrication of gold nanoantennae by X-ray interference lithography followed by focused ion beam milling. We then analyze their plasmon spectrum and compare it to similar structures manufactured by electron beam lithography. Conclusions are given in Sec. 4 .

\section{THEORY AND MODELING OF GOLD NANOANTENNAE}

\subsection{Fluorescence Enhancement}

For excitation power below saturation, the fluorescence signal $S_{o}$ for an isolated emitter is proportional to $\eta_{o}\left|\mathbf{d} \cdot \mathbf{E}_{o}\right|^{2}$, where $\eta_{o}$ is the quantum efficiency of the emitter, $\mathbf{d}$ is its electric dipole moment, and $\mathbf{E}_{o}$ is the excitation field at the position of the emitter. ${ }^{5} \eta_{o}$ is simply the ratio between the radiative decay rate $\gamma_{\mathrm{r}}^{o}$ and the total decay rate $\gamma_{\mathrm{t}}^{o}$. The total decay rate accounts also for a non-radiative decay $\gamma_{\mathrm{nr}}^{o}$, i.e. $\gamma_{\mathrm{t}}^{o}=\gamma_{\mathrm{r}}^{o}+\gamma_{\mathrm{nr}}^{o}$. When the emitter is coupled to a nanoantenna and we assume that the detection efficiency does not change, the fluorescence signal will now be proportional to $\eta|\mathbf{d} \cdot \mathbf{E}|^{2}$, where $\eta$ is the effective quantum efficiency that takes into account also the energy dissipated by the nanoantenna and $\mathbf{E}$ is the near field of the nanoantenna at the emitter position. Recalling that the nanoantenna modifies both the radiative decay rate, $\gamma_{\mathrm{r}}^{o} \rightarrow \gamma_{\mathrm{r}}$, and the total decay rate, $\gamma_{\mathrm{t}}^{o} \rightarrow \gamma_{\mathrm{t}}$, the effective quantum efficiency $\eta=\gamma_{\mathrm{r}} / \gamma_{\mathrm{t}}$ can be written as

$$
\eta=\frac{\eta_{o}}{\left(1-\eta_{o}\right) \gamma_{\mathrm{r}}^{o} / \gamma_{\mathrm{r}}+\eta_{o} / \eta_{\mathrm{a}}}
$$

where $\eta_{\mathrm{a}}$ is defined antenna quantum efficiency and corresponds to the fraction of energy dissipated solely by the nanoantenna and not by other intrinsic non-radiative channels, i.e. $\eta=\eta_{\mathrm{a}}$ when $\eta_{o}=1$. The fluorescence enhancement due to the nanoantenna is thus

$$
\frac{S}{S_{o}}=\frac{\eta}{\eta_{o}} \frac{|\mathbf{d} \cdot \mathbf{E}|^{2}}{\left|\mathbf{d} \cdot \mathbf{E}_{o}\right|^{2}} .
$$

Notice that the enhancement can be obtained either by increasing the electric field, by increasing the quantum efficiency or by both effects. The collection efficiency could also play a role, but we assume here that the system emitter-nanoantenna retains the dipole emission pattern of an isolated emitter.

It is well known that metal NPs exhibit a near field mostly radially polarized and particularly strong for excitation wavelengths close to the plasmon resonance. ${ }^{19}$ Therefore, achieving fluorescence enhancement by exploiting metal NPs could seem quite straightforward. However, one has to consider that the quantum efficiency decreases as the emitter approaches the $\mathrm{NP}^{17}$ so that there is a tradeoff between two competing effects. Consequently, to obtain strong fluorescence enhancements one has to design nanoantennae that do not quench the emitter.

Except for a single spherical NP, obtaining an analytical solution for the field enhancement and the decay rates can be extremely challenging. For this reason, we employ the finite-difference time-domain (FDTD) method to solve Maxwell's equations numerically. For the field enhancement we excite the NP with a broadband plane wave and compute the near field in selected positions after Fourier transforming the electric field in frequency domain. That allows us to compute the spectral dependence of the field enhancement within a single run. The calculation of the decay rates can be also performed within classical electrodynamics thanks to the equality between the normalized decay rates and the normalized power emitted by a classical dipole at the same position and orientation of the emitter, where for normalized quantities we intend decay rate and radiation with respect to the case without nanoantenna. ${ }^{20}$ 


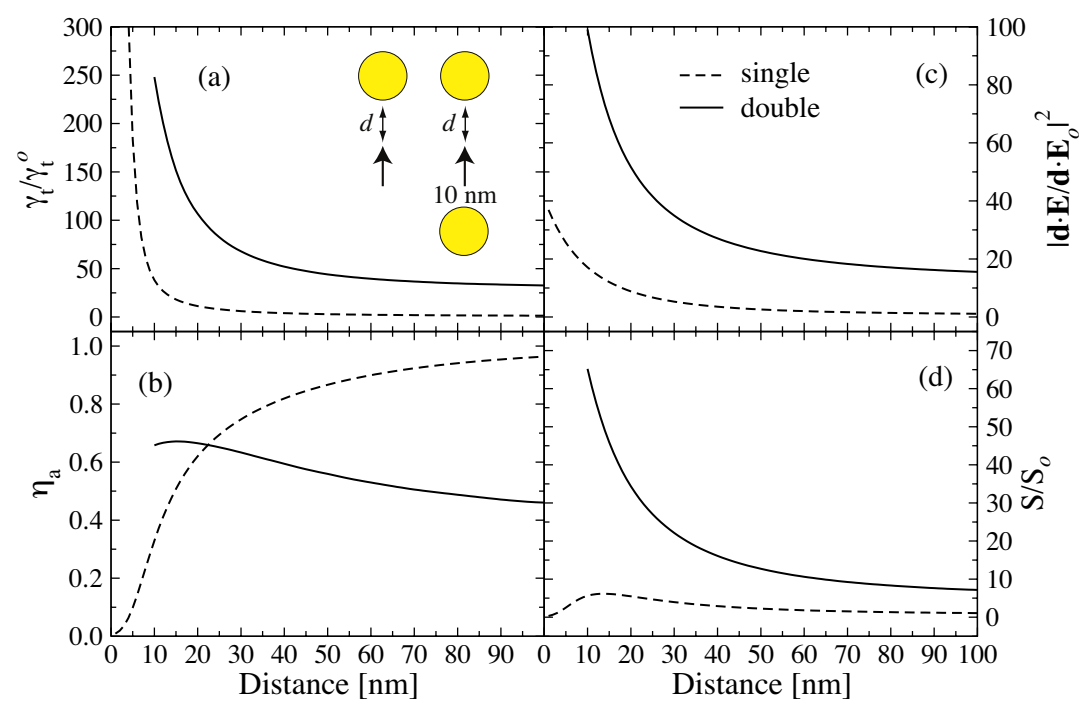

Figure 1. Normalized total decay rate $\gamma_{\mathrm{t}} / \gamma_{\mathrm{t}}^{o}(\mathrm{a})$, antenna quantum efficiency $\eta_{\mathrm{a}}(\mathrm{b})$, excitation enhancement $|\mathbf{d} \cdot \mathbf{E} / \mathbf{d} \cdot \mathbf{E}|^{2}$ (c) and fluorescence enhancement $S / S_{o}$ (d) for a single molecule coupled to one (single) or two (double) gold NPs. The inset describes the coupling scheme: molecule position and orientation, NPs position, distance $d$ from the molecule, which is varied from 10 to $100 \mathrm{~nm}$; for the two-NPs case, the one at the bottom is fixed at a distance of $10 \mathrm{~nm}$ from the molecule. Parameters: background medium is air, NPs radius $r=50 \mathrm{~nm}$, excitation wavelength $\lambda_{\mathrm{ex}}=532 \mathrm{~nm}$, emission wavelength $\lambda_{\mathrm{em}}=580 \mathrm{~nm}$.

\subsection{Single Molecule Coupled to One or Two Gold NPs}

Since it is already known that the field enhancement produced by two spherical metal NPs is larger than for a single one ${ }^{21}$ we first investigate if the same trend occurs also for the quantum efficiency The decay rates for two gold nanospheres have been been already studied in the literature, ${ }^{22}$ but without focusing on the quantum efficiency and on situations accessible to experiments. Moreover, instead of placing the emitter right midway from the two NPs, we think that a better comparison with the single NP case could be seen if the emitter were at fixed distance from one of the two NPs, while the other one is approached from far away.

The inset to Fig. 1 schematically shows how the coupling between emitter and nanoantenna is modified by changing the distance $d$. The molecule is oriented towards the NP and, for the case of two NPs, one of the two is fixed at a distance of $10 \mathrm{~nm}$ from the emitter. The gold ${ }^{23} \mathrm{NPs}$ have radius $r=50 \mathrm{~nm}$ and the background medium is air. The calculations are performed analytically for the single NP, 1,17,24 whereas the FDTD method is employed for the case of two NPs. The excitation wavelength chosen for computing the field enhancement is $\lambda_{\mathrm{ex}}=532 \mathrm{~nm}$, while the emission wavelength for the decay rates and the quantum efficiency is $\lambda_{\mathrm{em}}=580 \mathrm{~nm}$, which correspond to terrylene molecules in para-terphenyl (pT). ${ }^{5,25}$ Figure 1a shows the normalized total decay rate as a function of $d$ and for $\eta_{o}=1$. For distances of the order of $100 \mathrm{~nm}$, the total decay rate for the emitter coupled to two NPs is almost equal to the one coupled to a single NP when the distance is $10 \mathrm{~nm}$. Indeed, the curve corresponding to a single NP shows that the modification of the decay rate begins to be significant only for distances smaller than $30 \mathrm{~nm}$. When both NPs are close to the emitter, the decay rate is clearly larger than for a single NP. Figure 1b shows the antenna quantum efficiency for one and two NPs. For one NP the quantum efficiency drops quickly to zero when the distance becomes smaller than $20 \mathrm{~nm},{ }^{5,17}$ whereas for two NPs the quantum efficiency slightly increases up to $d \simeq 15 \mathrm{~nm}$ and then starts decreasing until quenching the emitter (not shown), but at shorter distances than for the previous case. Once again, when the second NP is far away the quantum efficiency corresponds to the case of a single NP at $10 \mathrm{~nm}$ from the emitter.

Figure 1c displays the excitation enhancement as a function of the NP distance. Again, the near field is stronger when the two NPs are close than for the case of a single one. By combining the data from Figs. 1b and $1 \mathrm{c}$ one can understand the competing effects of intensity enhancement and quantum efficiency. It is evident that the equilibrium between them is different for one NP and two NPs. That is even more apparent in Fig. 1d, where 


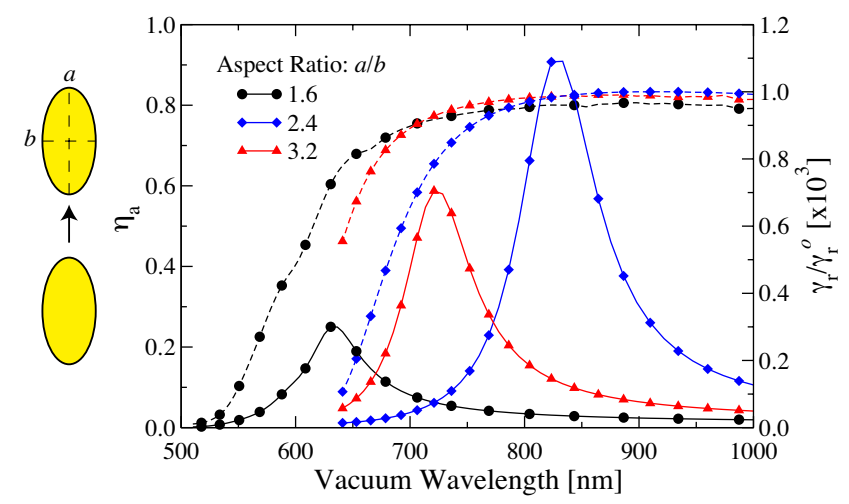

Figure 2. Quantum efficiency $\eta_{\mathrm{a}}$ (dashed lines) and normalized radiative decay rate $\gamma_{\mathrm{r}} / \gamma_{\mathrm{r}}^{o}$ (solid lines) as a function of wavelength for three gold nanoantennae having the same volume but different aspect ratio $(a / b)$. Parameters: aspect ratio $1.6(a=76 \mathrm{~nm}, b=48 \mathrm{~nm})$, aspect ratio $2.4(a=100 \mathrm{~nm}, b=42 \mathrm{~nm})$, aspect ratio $3.2(a=122 \mathrm{~nm}, b=38 \mathrm{~nm})$. The gap size is $20 \mathrm{~nm}$ and the background index is equal to 1.3 for all nanoantennae. The molecule is positioned in the middle of the gap and aligned as shown in the figure.

the fluorescence enhancement, see Eq. 2, is plotted for the two situations assuming that the emitter has initial quantum efficiency $\eta_{o}$ equal to one. Notice that while the fluorescence enhancement achievable with a single NP is maximal at a distance of approximately $14 \mathrm{~nm}$, with a nanoantenna made of two NPs the enhancement is still increasing when the emitter is $10 \mathrm{~nm}$ from both NPs. Therefore, in a well controlled experiment one should be able to see almost an order of magnitude improvement with respect to the single NP studied in previous works. ${ }^{5,6}$ One has to keep in mind that the coupling of the two NPs shifts the plasmon resonance towards longer wavelengths ${ }^{21,28}$ thus making the overall enhancement increasing less rapidly than if the emitter could stay tuned to the plasmon resonance.

\subsection{Optimization of Gold Nanoantennae}

Fig. 1 shows that it is better to use two NPs instead of one, however one can ask if there are ways to achieve even stronger fluorescence enhancements by careful design of the nanoantenna. For example, it has been shown that the so-called bow-tie nanoantenna ${ }^{28}$ and nanorod pairs ${ }^{29}$ exhibit a strong field enhancement in the proximity of the gap between the two NPs. A large intensity enhancement can also be obtained at the apex of spheroidal NPs. ${ }^{30}$ Nevertheless, can one at the same time preserve a high quantum efficiency? Within the electrostatic approximation, Gersten and Nitzan have shown that single spheroidal silver NPs provide a high quantum efficiency even when the emitter is very close to the metal surface. ${ }^{31}$ Very recently, we have demonstrated that this is true also for single and double gold spheroids using full electrodynamics calculations. ${ }^{18}$ In particular, we have shown that our designed nanoantennae yield very large radiative decay rates (up to three order of magnitudes) while $\eta_{\mathrm{a}}$ stays above 0.8 in the near infrared spectral range. By invoking reciprocity we can state that also a very strong near field enhancement should occur, and in fact that is what we have found by performing additional calculations.

To perform experiments on single molecules we typically work with terrylene and dibenzanthanthrene (DBATT) molecules embedded in pT crystals, since they are highly photostable and well oriented. ${ }^{25-27}$ Because their emission spectrum is around $600 \mathrm{~nm}$, we have to design a nanoantenna resonant in that wavelength range. Spheroidal NPs have the advantage that the plasmon resonance can be easily tuned to the wavelength of interest by changing their aspect ratio. ${ }^{30,32}$ In Fig. 2 we show how one can shift the plasmon resonance from the near infrared to the visible range using pairs of gold spheroidal NPs separated by a gap of $20 \mathrm{~nm}$. Unfortunately, as the aspect ratio decreases, the decay rate enhancement also decreases since the NP becomes less sharp. The same trend occurs for the field enhancement. ${ }^{30}$ For the calculations of Fig. 2 the background index is set to 1.3 , corresponding to an effective index between glass (1.52) and air to take into account that in a fabricated sample the nanoantennae are lying on a glass cover slip. The nanoantenna with aspect ratio 1.6 is resonant close to the excitation and emission wavelengths of DBATT in pT $\left(\lambda_{\mathrm{ex}}=615 \mathrm{~nm}\right.$ and $\left.\lambda_{\mathrm{em}}=630 \mathrm{~nm}\right){ }^{27}$ Notice that 

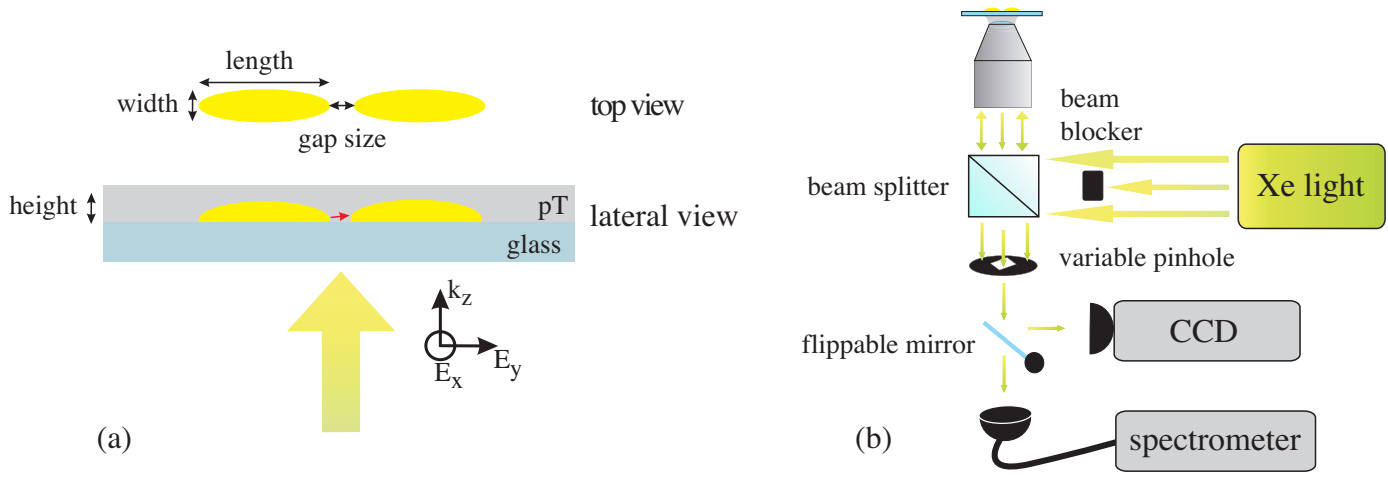

Figure 3. Sample layout (a) and optical characterization setup (b). Gold nanoantennae are fabricated on a glass cover slip and the molecules can be added by spin coating a thin film of pT doped with DBATT molecules. Notice the beam blocker and the variable pinhole in (b) to block the background light end select only the signal coming from a single nanoantenna.

for terrylene molecules one should further reduce the aspect ratio to reach nearly the spherical shape discussed in Fig. 1, because excitation and emission occur at shorter wavelengths $\left(\lambda_{\mathrm{ex}}=532 \mathrm{~nm}\right.$ and $\left.\lambda_{\mathrm{em}}=580 \mathrm{~nm}\right) .^{5}$ Furthermore, the quantum efficiency cannot be as high as for the infrared case due to the strong absorption of gold for wavelengths shorter than $600 \mathrm{~nm}$. These practical considerations lead us to the fabrication of gold nanoantennae designed for the spectral region around 600-650 nm for DBATT molecules. Lastly, Fig. 2 clarifies that the antenna quantum efficiency is almost independent of the aspect ratio in the infrared range, contrary to what happens at wavelengths where gold starts to have more absorption. ${ }^{23}$

\section{FABRICATION AND CHARACTERIZATION OF GOLD NANOANTENNAE}

The nanoantennae described in the previous section can be successfully manufactured taking advantage of stateof-the-art fabrication methods and instruments like focus ion beam (FIB) milling and electron beam lithography (EBL). Even if both FIB and EBL approaches allow the design of features with nanometric resolution, these techniques present different advantages and disadvantages with respect to each others that should be considered in order to optimize the fabrication step. Briefly, EBL is a parallel method, i.e several, nominally identical, structures can be fabricated in the same exposure, while FIB is serial one. On the contrary EBL is usually followed by an evaporation and lift-off step. In particular, this last step results to be particularly difficult when the dimensions of the structures are below $50 \mathrm{~nm}$. On the other hand, FIB lithography is typically a single-step process where the wanted structures are directly shaped out of a material.

\subsection{Experimental Setup}

Figure 3a shows a sketch of the samples that could be manufactured on a glass substrate. The glass interface makes impossible achieving a perfectly spheroidal shape. For this reason, we have performed additional calculation to take into account these modifications to the nanoantenna original design. With both fabrication methods one can select the width, the length, the height of the NPs and the gap between the two. Once the nanoantenna has been manufactured, a solution containing pT doped with DBATT molecules is spin coated on the sample. Subsequently, a pT crystal embedding DBATT molecules aligned nearly parallel to the glass interface should form. ${ }^{27}$ The position and azimuthal orientation of molecule is left to chance, which is small considering the area of the nanoantenna gap. The sample is illuminated through the glass cover slip with light polarized along the nanoantenna short or long axes.

Before studying the fluorescence enhancement, we have focused on the optical characterization of nanoantennae by measuring their plasmon spectrum without spin coating the pT film. Figure 3b sketches the optical setup for illumination and detection. We employ a white light source which is focused on the sample by an inverted microscope. The polarized light illuminates the nanoantennae by total internal reflection. Concerning the detection scheme, a variable pinhole collects only the scattered light coming for the direction perpendicular 

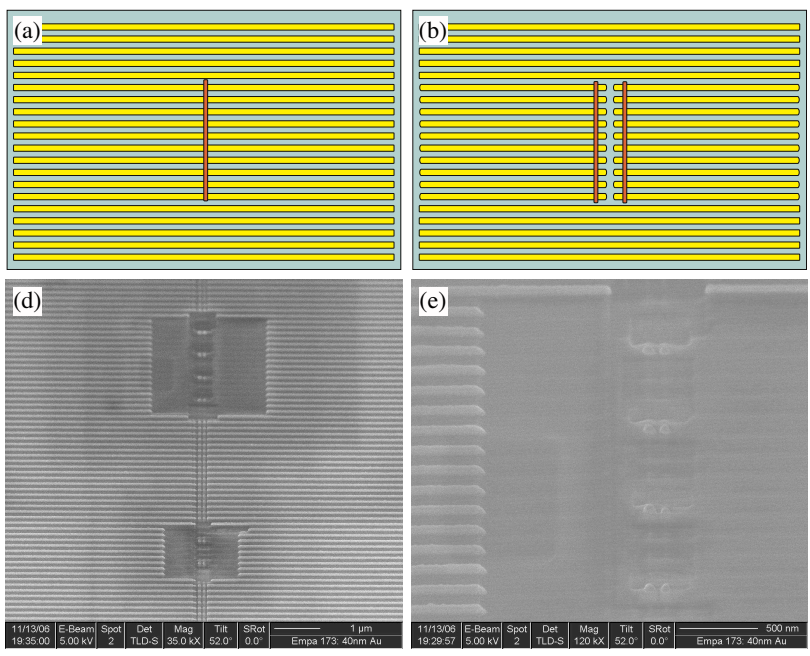
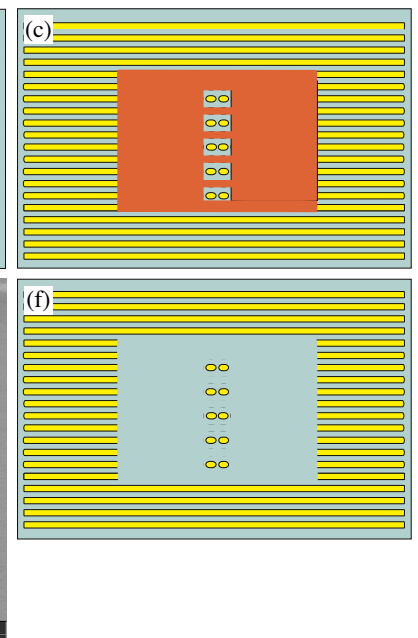

Figure 4. Gold nanoantenna fabrication using XIL and FIB milling. (a) One cut is made in the gold grating to define the gap size of the nanoantenna; (b) two other cuts are made to define the length of the two NPs composing the nanoantenna; (c) the gold surrounding the nanoantennae is removed; (f) an array of gold nanoantennae is finally obtained. (d) and (e) are SEM images of the fabricated nanoantennae.

to the glass film, i.e. where the incident light is strongly suppressed by the beam blocker present in the illumination path. A movable mirror can redirect light to a couple-charged-device (CCD) camera for imaging and to a spectrometer for collecting the plasmon spectrum. Moreover, by changing the size of the pinhole it is possible to see only one nanoantenna or several nanoantennae per image.

\subsection{Gold Nanoantennae Fabricated using FIB}

In the case of FIB, the nanoantennae are shaped out of a gold grating patterned using X-ray Interference Lithography (XIL). ${ }^{33}$ Taking advantage of this technique, we patterned a commercial microscope cover slide with gold stripes (width $40 \mathrm{~nm}$, height $40 \mathrm{~nm}$ ). This step considerably reduces the milling time for the nanoantenna fabrication since high resolution (low milling speed) is only needed for the central cut while the rest can be done with low resolution. The nanoantennae were fabricated on a FEI Strata 235 dual-beam machine. Ions used were $\mathrm{Ga}^{+}$extracted from a liquid metal ion source and the acceleration potential was $30 \mathrm{kV}$. To fabricate the central gap, see Fig. 4a, the ion current was set to the lowest available value of $1 \mathrm{pA}$, which corresponds to an ion beam with a full-width at half-maximum (FWHM) of $7 \mathrm{~nm}$. Single pixel lines were sputtered at normal incidence using a raster scan strategy with a pixel spacing of $6.5 \mathrm{~nm}$ and a total dwell time per pixel of $22 \mathrm{~ms}$ achieved with 8 scan repetitions. Minimum gap width on the glass substrate was $15 \mathrm{~nm}$, determined by scanning electron microscopy (SEM). Subsequently, two lateral cut were performed to defined the NP length, see Fig. $4 \mathrm{~b}$. Lastly, the surrounding gold was removed using high milling speed, see Fig. 4c. The final result, sketched in Fig. 4f, is a series of gold nanostructures isolated from the gold grating. Figures 4d and 4e show SEM pictures of nanoantennae fabricated by this method. In both figures the gold stripes produced by XIL are still visible.

The gold nanoantennae have been characterized using the setup described in Fig. 3b. Figure 5b shows a CCD image of nanoantennae. In a second step, the pinhole is gradually closed until only the light from a single nanoantenna is collected and sent to the spectrometer. The plasmon spectrum for polarization along the nanoantenna long axis is shown in Fig. 5c, which corresponds to the nanoantenna displayed in the SEM image of Fig. 5a. The plasmon resonance is quite broad probably because the FIB milling did not remove completely the gold grating, as one can see from the SEM image. Moreover, even if some intermediate gold lines were removed, the nanoantennae are still closely packed so that the pinhole is not able to completely isolate the light coming from a single nanoantenna. Since removing a lot more grating is time-consuming and since a second-step milling to eliminate the residual gold could irremediably damage the nanostructure, we have looked for an alternative approach to fabricated them, namely EBL. 

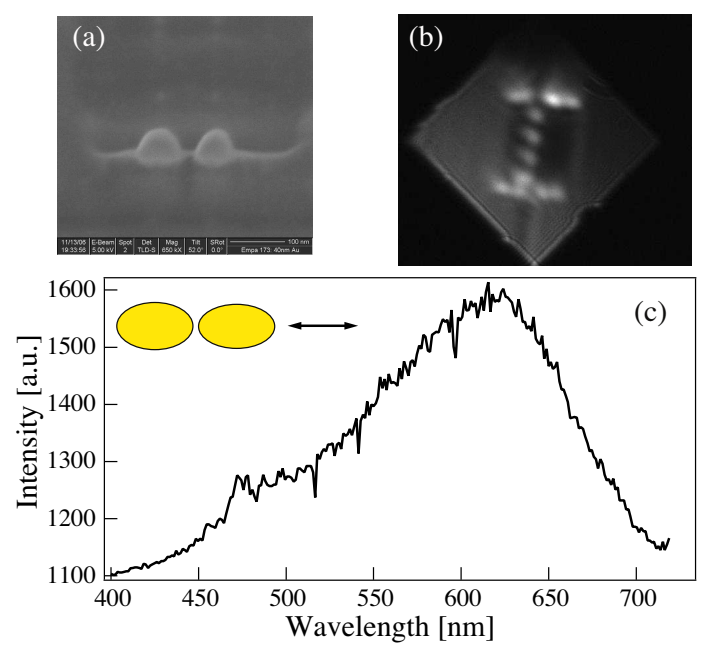

Figure 5. Optical characterization of gold nanoantennae fabricated by XIL and FIB milling. (a) SEM image of a single nanoantenna; (b) dark field image of nanoantennae; (c) plasmon spectrum of the single nanoantenna shown in (a) for polarization in the long axis. Nanoantenna dimensions: gap $42 \mathrm{~nm}$, length 75 and 58 respectively, width $40 \mathrm{~nm}$ and height $25 \mathrm{~nm}$.

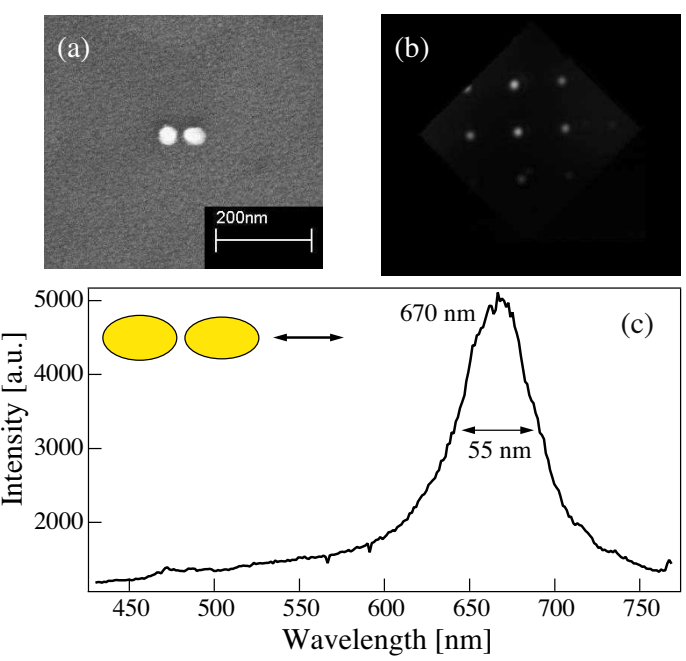

Figure 6. Optical characterization of gold nanoantennae fabricated by EBL. (a) SEM image of a single nanoantenna; (b) dark field image of nanoantennae; (c) plasmon spectrum of the single nanoantenna shown in (a) for polarization in the long axis. Nanoantenna dimensions: gap $15 \mathrm{~nm}$, length $40 \mathrm{~nm}$ for both NPs, width $35 \mathrm{~nm}$ and height $40 \mathrm{~nm}$.

\subsection{Gold Nanoantennae Fabricated using EBL}

With EBL we can fabricate very many nanoantennae with the desired spacing, thus increasing the chance of localizing the molecule at the right place yet having the certainty of imaging a single nanoantenna. The structure parameters were again chosen to have a plasmon resonance around $600-650$ nm. Figure $6 \mathrm{~b}$ displays a CCD image of an array of nanoantennae manufactured by EBL, while Fig. 6c shows the plasmon spectrum measured for the single nanoantenna whose SEM picture is given in Fig. 4a. The spectrum exhibits a resonance centered around $670 \mathrm{~nm}$, a bit red-shifted with respect to the target design, with $55 \mathrm{~nm}$ FWHM. The nanoantennae are now sufficiently spaced to guarantee that the spectrum belongs to a single structure. 


\subsection{Discussion}

The results of Fig. 6 in comparison to those of Fig. 5 evidence that the EBL approach is more suitable for the fabrication of nanoantennae since it can supply very many nanoantenna having the desired spectral and structural features. The next step is to add the molecules by spin coating the pT film doped with DBATT. It should be emphasized that, even if the nanoantennae where successfully fabricated, it is very challenging to place an emitter exactly in the middle of the structure and with the right orientation. Indeed theoretical calculations have shown that the strong enhancement occurs only if the emitter is within a few tens of nanometers from the gap and oriented parallel to the nanoantenna long axis. ${ }^{32}$ For this reason, if we do not want to count exclusively on chance, we should developed a technique to place and orient the emitter more deterministically. For instance, surface chemistry might be helpful in performing this task. ${ }^{7}$

\section{CONCLUSIONS}

We have theoretically studied the fluorescence enhancement of a single emitter coupled to a nanoantenna made of two gold spheroidal NPs. We have shown that there is a qualitative and quantitative difference between coupling a single emitter to one or two spherical NPs if the distance between the emitter and one NP is varied. To perform experiments on single molecules we have tuned the plasmon resonance close to the excitation and emission wavelengths of highly photostable molecules starting from our optimized designs for the infrared spectral range. We have fabricated gold nanoantennae using two different approaches, namely XIL followed by FIB milling and EBL. From the SEM pictures and the optical characterization we have found that the second approach offers better results, especially considering the fact that very many nanoantennae with identical properties have to be fabricated to increase the chance of placing the emitter in the right position. Since the photostability of our molecules strongly depends on the quality of the pT crystal, we are currently studying how the crystal forms in the presence of gold. For this reason and the issue of localizing the molecule in a very small region, we are also trying different approaches that would allow us to study the interaction of a single molecule with two NPs.

\section{ACKNOWLEDGMENTS}

The gold gratings were fabricated at Paul Scherrer Institute and FIB milling was performed at EMPA. The EBL of gold nanoantennae was performed at FIRST, the Center for Micro- and Nanoscale Science at ETH Zurich. We are grateful to U. Håkanson, H. Eghlidi, A. Mohammadi, and N. Mojarad for fruitful discussions and to S. Meier for operating the FIB. This work was financed by the ETH Zurich initiative on Composite Doped Metamaterials $(\mathrm{CDM})$.

\section{REFERENCES}

1. C. F. Bohren, and D. R. Huffman, Absorption and scattering of light by small particles, John Wiley and Sons, New York, 1983.

2. U. Kreibig, and M. Vollmer, Optical properties of metal clusters, Springer, Heidelberg, 1995.

3. J. R. Lakowicz, "Radiative decay engineering 5: metal-enhanced fluorescence and plasmon emission," Anal. Biochem. 337, pp. 171-194, 2005.

4. J. N. Farahani, D. W. Pohl, H.-J. Eisler, and B. Hecht, "Single quantum dot coupled to a scanning optical antenna: a tunable superemitter," Phys. Rev. Lett. 95, pp. 017402(4), 2005.

5. S. Kühn, U. Håkanson, L. Rogobete, and V. Sandoghdar, "Enhancement of single-molecule fluorescence using a gold nanoparticle as an optical nanoantenna," Phys. Rev. Lett. 97, pp. 017402(4), 2006.

6. P. Anger, P. Bharadway, and L. Novotny, "Enhancement and quenching of single-molecule fluorescence," Phys. Rev. Lett. 96, pp. 113002(4), 2006.

7. J. Zhang, Y. Fu, M. H. Chowdhury, and J. R. Lakowicz, "Metal-enhanced single-molecule fluorescence on silver particle monomer and dimer: coupling effect between metal particles," Nano Lett. 7, pp. 2101-2107, 2007.

8. P. J. Schuck, D. P. Fromm, A. Sundaramurthy, G. S. Kino, and W. E. Moerner, "Improving the mismatch between light and nanoscale objects with gold bowtie nanoantennas," Phys. Rev. Lett. 94, pp. 017402(4), 2005. 
9. M. Danckwerts, and L. Novotny, "Optical frequency mixing at coupled gold nanoparticles," Phys. Rev. Lett. 98, pp. 026104(4), 2007.

10. E. Cubukcu, E. A. Kort, K. B. Crozier, and F. Capasso, "Plasmonic laser antenna," Appl. Phys. Lett. 89, pp. 093120(3), 2006.

11. K. A. Willets and R. P. Van Duyne, "Localized surface plasmon spectroscopy and sensing," Ann. Rev. Phys. Chem. 58, pp. 267-297, 2007.

12. M. Moskovits, "Surface-enhanced spectroscopy," Rev. Mod. Phys. 57, pp. 783-826, 1985.

13. S. Nie, and S. R. Emory, "Probing single molecules and single nanoparticles by surface-enhanced Raman scattering," Science 275, pp. 1102-1106, 1997.

14. P. Mühlschlegel, H.-J. Eisler, O. J. F. Martin, B. Hecht, and D. W. Pohl, "Resonant optical antennas," Science 308, pp. 1607-1609, 2005.

15. J. S. Biteen, D. Pacifici, N. S. Lewis, and H. A. Atwater, "Enhanced radiative emission rate and quantum efficiency in coupled silicon nanocrystal-nanostructured gold emitters," Nano Lett. 5, pp. 1768-1773, 2005.

16. H. Mertens, and A. Polman, "Plasmon-enhanced erbium luminescence," Appl. Phys. Lett. 89, pp. 211107(3), 2006.

17. R. Ruppin, "Decay of an excited molecule near a small metal sphere," J. Chem. Phys. 76, pp. 1681-1684, 1982 .

18. L. Rogobete, F. Kaminski, M. Agio, and V. Sandoghdar, "Design of nanoantennae for the enhancement of spontaneous emission," Opt. Lett. 32, pp. 1623-1625, 2007.

19. B. J. Messinger, K. U. von Raben, R. K. Chang, and P. W. Barber, "Local fields at the surface of noble-metal microspheres," Phys. Rev. B. 24, pp. 649-657, 1981.

20. F. Kaminski, V. Sandoghdar, and M. Agio, "Finite-difference time-domain modeling of decay rates in the near field of metal nanostructures," J. Comput. Theor. Nanosci. 4, pp. 635-643, 2007.

21. P. K. Aravind, A. Nitzan, and H. Metiu, "The interaction between electromagnetic resonances and its role in spectroscopic studies of molecules adsorbed on colloidal particles or metal spheres," Surf. Sci. 110, pp. 189204, 1981.

22. L. A. Blanco, F. J. García de Abajo, "Spontaneous light emission in complex nanostructures," Phys. Rev. B 69, pp. 205414(12), 2004.

23. CRC Handbook of Chemistry and Physics, 2006 (eighty-seventh edition). http://www.hbcpnetbase.com/.

24. V. V. Klimov, M. Ducloy, and V. S. Letokhov, "Radiative frequency shift and linewidth of an atom dipole in the vicinity of a dielectric microsphere," J. Mod. Opt. 43, pp. 2251-2267, 1996.

25. R. J. Pfab, J. Zimmermann, C. Hettich, I. Gerhardt, A. Renn, and V. Sandoghdar, "Aligned terrylene molecules in a spin-coated ultrathin crystalline film of p-terphenyl," Chem. Phys. Lett. 387, pp. 490-495, 2004.

26. A.-M. Boiron, B. Lounis, and M. Orrit, "Single molecules of dibenzanthanthrene in $n$-hexadecane," J. Chem. Phys. 105, pp. 3969-3975, 1996.

27. I. Gerhardt, G. Wrigge, P. Bushev, G. Zumofen, M. Agio, R. Pfab, and V. Sandoghdar, "Strong extinction of a laser beam by a single molecule," Phys. Rev. Lett. 98, pp. 033601(4), 2007.

28. A. Sundaramurthy, K. B. Crozier, G. S. Kino, D. P. Fromm, P. J. Schuck, and W. E. Moerner, "Field enhancement and gap-dependent resonance in a system of two opposing tip-to-tip Au nanotriangles," Phys. Rev. B 72, pp. 165409(6), 2005.

29. J. Aizpurua, G. W. Bryant, L. J. Richter, F. J. García de Abajo, B. K. Kelley, and T. Mallouk, "Optical properties of coupled metallic nanorods for field-enhancement spectroscopy," Phys. Rev. B 71, pp. 235420(13), 2005.

30. N. Calander, and M. Willander, "Theory of surface-plasmon resonance optical-field enhancement at prolate spheroids," J. Appl. Phys. 92, pp. 4878-4884, 2002.

31. J. Gersten, and A. Nitzan, "Spectroscopic properties of molecules interacting with small dielectric particles," J. Chem. Phys. 75, pp. 1139-1151, 1981.

32. L. Rogobete, F. Kaminski, A. Mohammadi, M. Agio, and V. Sandoghdar, in preparation.

33. H. H. Solak, Y. Ekinci, P. Käser, and S. Park, "Photon-beam lithography reaches $12.5 \mathrm{~nm}$ half-pitch resolution," J. Vac. Sci. Technol. B 25, pp. 91-95, 2007. 